\title{
Dynamic Representations of Spatial Events - The Example of a Typhoon
}

\author{
Yufeng He $\mathrm{e}^{\mathrm{a}, \mathrm{b}, \mathrm{c}, \mathrm{d}}$ (corresponding author), Barbara Hofer ${ }^{\mathrm{d}}$, Yehua Sheng ${ }^{\mathrm{a}, \mathrm{b}, \mathrm{c}}$, Yi Huang \\ 181301016@njnu.edu.cn, barbara.hofer@sbg.ac.at, shengbyehua@njnu.edu.cn, huangyi@njupt.edu.cn
}

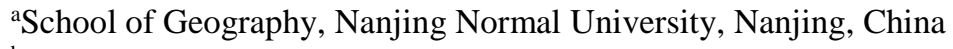

${ }^{\mathrm{b}}$ Key Laboratory of Virtual Geographic Environment (Nanjing Normal University), Ministry of Education, Nanjing, China

${ }^{\mathrm{c} J i a n g s u}$ Center for Collaborative Innovation in Geographical Information Resource Development and Application, Nanjing, China

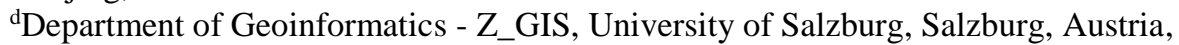

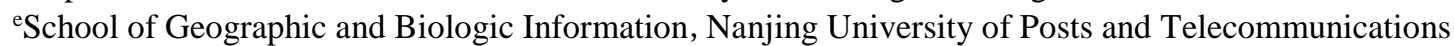

\begin{abstract}
The Geographic scene is a conceptual model that provides a holistic representation of the environment. This model has been developed in order to overcome limitations of geographic information systems (GIS) concerning interactions between features and the representation of dynamics. This contribution translates the theoretical model into an implementation of a dynamic data model in the graph database $\mathrm{Neo} 4 \mathrm{j}$ and applies it to GIS data representing the dynamic information of a typhoon. The specific focus of the contribution is on choices made in the process of generation of the implementation of the example and the potential queries it supports.
\end{abstract}

Keywords: spatio-temporal dynamics; geographic scene data model; spatial process; spatial event; graph database

\section{Introduction}

While conventional data models used in geographic information systems (GIS) can describe the states or changes of individual objects or cells, these models do not portray the internal mechanisms of geographic change(Hornsby and Cole, 2007). Ample approaches to extend GIS with capabilities to represent and analyse time and dynamics exist (Cao et al., 2018; Li et al., 2009; Uschold, 2008; Zhao and Li, 2009; Galton); yet many of them lack practical application.

This paper addresses this weakness and presents a reallife case study of a typhoon event that makes use of a dynamic data model implemented in the graph database Neo4j. The dynamic data model is based on the conceptual model of geographic scenes. The conceptual model allows for the identification of events, processes and states and their relationships and thereby lies the foundation for the implemented dynamic data model.

As a carrier and container for the existence, occurrence and development of geographic elements, geographic phenomena and geographic event(Huang et al., 2019), the geographic scene is a holistic model based on human cognition that has a system theoretical view on the interaction between scene objects such as people, objects, events, phenomena(Lü et al., 2019; Lu et al., 2018).

Taking the conceptual model as starting point, this work first identifies relationships and interactions between processes, events and states in geographic scenes. These relationships are then translated into a data model that is implemented in the graph database Neo4j. The resulting implementation allows querying of process and event related questions as demonstrated for the application case of typhoon Lekima that severely hit several places in China in 2019. The following sections summarize the conception and implementation of the dynamic data model.

\section{Conception of the Dynamic Data Model}

The primary elements of the dynamic data model are events, processes and states. For every representation of dynamics of a geographic scene, these elements need to be identified and their relationships established. Thus, the main elements of the spatio-temporal framework providing the foundation of the dynamic data model are: geographic scenes with a focus on a specific phenomenon, events, processes and states (Fig. 1). Definitions are provided in the following. 


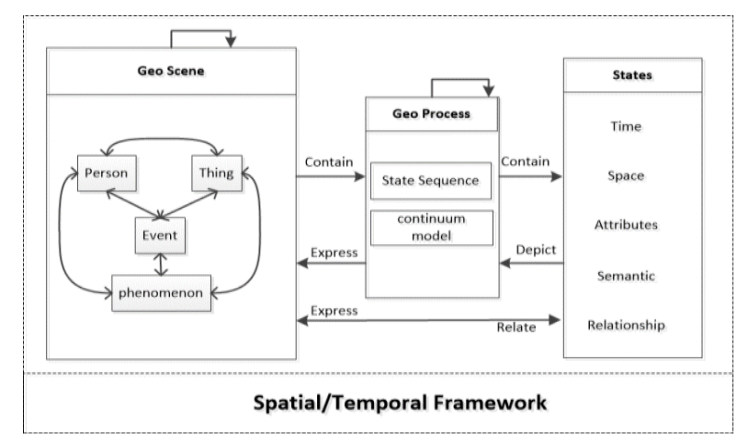

Figure 1: Objects and interactions of the geographic scene model.

\subsection{Definition of Phenomenon, Event, Process and State}

Definition 1 - phenomenon: Geographic phenomenon is the existence or occurrence of something that cannot be identified as an entity that is continuously distributed and changing within a certain range in a geographic scene. Distinguished from the geographic feature with boundary determinism and discrete nature, geographic phenomenon refers to the continuous distribution and blurred form in the geographic scene, such as sandstorm, tropical cyclone, etc.

Definition 2 - events: A geographical event is a thing, act, or fact that occurs suddenly at a specific time and in a geographical scene. It generally refers to something with a considerable influence that has happened or is happening.

Definition 3 - process: Geographic process refers to the trajectory of the characteristics (location, geometry, semantics, properties, interrelationships, etc.) of a geographic object or phenomenon as it changes over time.

Definition 4 - state: State is the instantaneous characteristics (semantics, location, attributes, and relationships) that are realized at a particular moment. In the traditional GIS data model, state refers specifically to the instantaneous characteristics of geographic features, and state is also the main way to portray the real world; while in geographic scenes, the state is the instantaneous characteristics of events, processes, and phenomenal in geographic scenes.

\subsection{The Relationship between Geographic Processes, Events and States}

Both geographic events and geographic processes are capable of modeling geographic change within a finite period. Events are bounded and discrete from each other, but processes within events are continuous. A geographic event is a discrete occurrence intrinsically bounded (Galton, 2015), which can be conceptualized as a point occurrence at a coarse time scale. Geographic events focus on historical time descriptions of occurrences with sudden changes and significant events with identifiable characteristics. Geographic processes are continuous with focus on what is happening, or what is continuing to happen at a given time.

Events can be composed of lower granularity temporally non-intersecting subevents. Since processes are used to represent the trajectory of change within an event, events also can be aggregated from many sub-processes that are independent of each other without overlapping. Geographic events express sudden changes in the scene and geographic processes describe gradual changes in the scene. When a geographic significant process occurred in the past, the process can be abstracted as an event, as shown in Fig.2.

For example, a geohazard event may include the occurrence of an earthquake hazard event followed by a landslide or mudslide event. The geohazard is a geographical event, and earthquake and landslide are subevents of the geographical event. If the granularity of investigation of events changes and a detailed view of an event is considered, an event can also be viewed as a process that has certain phases of its own. The differentiation between events and processes is related to temporal granularity of the investigation.

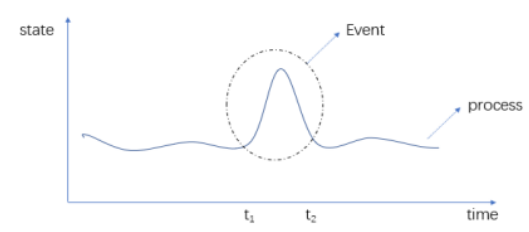

Figure 2: Events are sudden and significant processes

The occurrence and development of an event must have a geographical process or a series of geographical processes corresponding to it while the process is not certainly a geographical event that existed.

Geographic event types are implicit in the geographic state and that geographic events erupt when the state of a geographic scene feature reaches a threshold. The occurrence of a geographic event initializes, terminates, and changes the state of the scene object, and also causes changes in geographic processes and events. Geographic processes can maintain or change the geographic state, while the relationship among geographic processes is mutual influence or independent parallelism. The relationship between events, processes, and states is shown in Fig.3. 


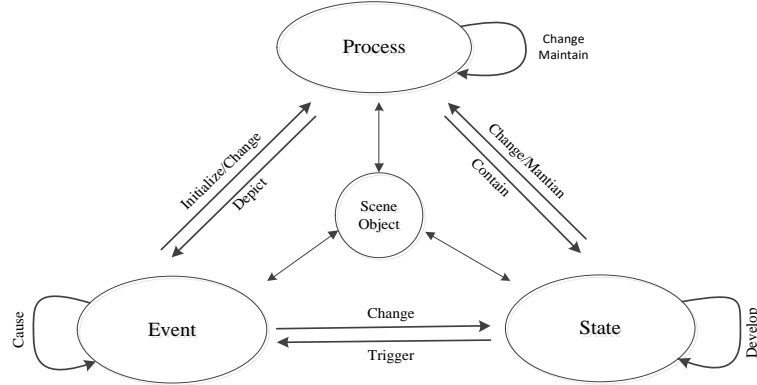

Figure 3: The relationship among event, process, state, and scene object

\section{Implementation of the Dynamic Data Model exemplified with Typhoon}

\subsection{Typhoon Dynamic Model}

To construct the evolutionary model, it is necessary to sort out the various relationships involved in the typhoon dynamic geographic processes, and then to build an event-process-centered state sequence model to represent spatio-temporal changes in the geographic scene. As we know, a typhoon is a sudden geographic event that causes major impacts and disasters. To detail the behavior of the typhoon, the typhoon process was defined to express dynamic changes and how the typhoon develops. The state of a typhoon is its instantaneous shape, which is described as wind circle, direction, central pressure, latitude, longitude, speed, and time. The typhoon's spatial and temporal changes were simulated by the state sequence. Typhoon processes can generally be divided into four stages with different semantics: birth, development, keeping, and vanishing stage (shown in fig. 4).

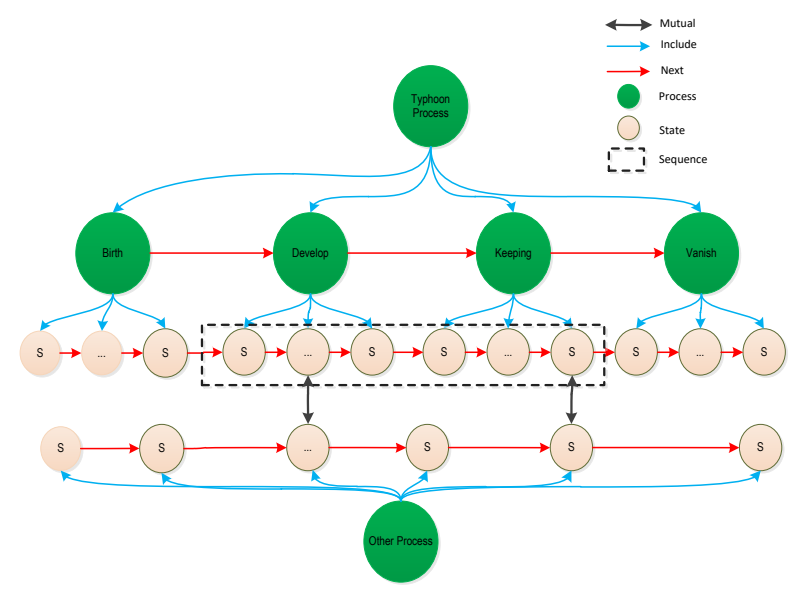

Figure 4: Typhoon dynamic process model structure

Three aspects depict the typhoon process' inner relationship: (1) hierarchy relationship using the relationship between processes and states. (2) developmental relationships consisting of states and states, processes and processes. (3) correlation relationship between events-processes, events-states to establish the spatio-temporal evolution rules. Inclusion relations include processes containing sub-processes, processes containing state sequences, state sequences containing states, and processes containing states. The development relation is the evolution of the process in time, expressing the progression between sub-processes and sub-processes, and between states and states, and they are expressed as sequential relations in time (Precede and Next). The interrelationship, mainly contains causality, subordination, interaction, and spatial relationship. The UML model shows the relationship among events, processes, and states as well as spatio-temporal development with object-oriented thinking (Fig. 5).

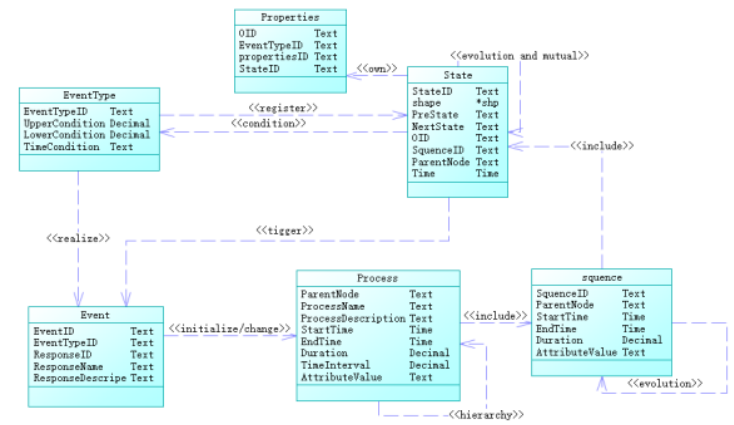

Figure 5: UML model of events, processes and states of the geographic scene

Viewing the coastal area of China as a geographic scene, a cumulative total of 492 typhoon events occurred in the scene since 1949 and formed 631 landfall events. In this paper, Lekima (No. 1909), the largest typhoon in 2019, was selected as a case to study, and the Lekima typhoon trajectory consisted of 160 typhoon monitoring points provided by China Weather Typhoon Network, as shown in Fig. 6.

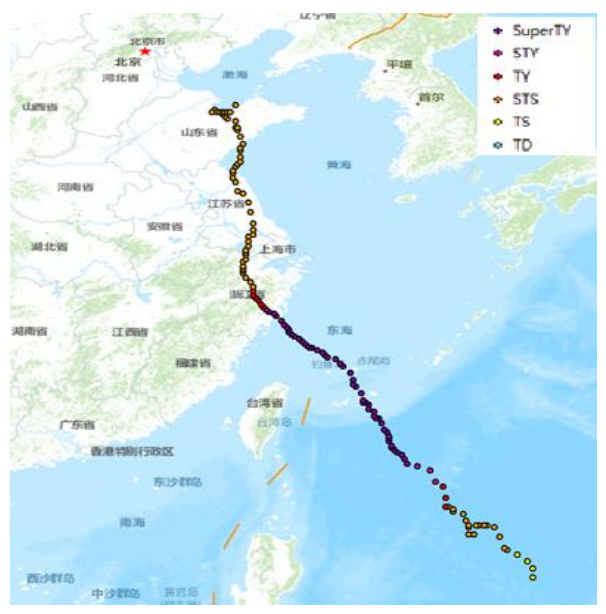


Figure 6: Typhoon Lekima intensity and trajectory. According to the China Meteorological Administration GBT 19201-2006 notice, tropical cyclones are divided into six classes: Super Typhoon (Super TY), Severe Typhoon (STY), Typhoon, Severe tropical storm (STS), Tropical storm (TS), Tropical depression (TD).

Combining typhoon disaster information in Taizhou City and typhoon warning information by Zhejiang Meteorological Bureau, the data was organized and stored according to the geographic scene conceptual model to elaborate the spatio-temporal evolution of typhoon Lekima. The Lekima typhoon event is the largest event, which contains the Lekima invasion of Taizhou as well as the landfall event. Each typhoon event was associated with typhoon processes by "depicted by" edges. Thus, Lekima Typhoon process describes the dynamics of the typhoon Lekima event in detail. The process of invasion of the typhoon into Taizhou details the Taizhou invasion event. In addition, building the interrelationship between the warning status and the typhoon status at the same moment to provide a link between the typhoon status and the warning status facilitates the organization of information for subsequent typhoon studies. In terms of geographic scene features, features of the typhoon Lekima scene involved human victims, things such as transportation facilities, communication facilities, electric power facilities, farmland, houses, enterprises, natural disasters, and other objects affected by the typhoon.

\subsection{Typhoon Event Construction Method}

Based on the UML model structure of event, process and state, combined with the properties of the typhoon, we organized the typhoon states information, typhoon subprocesses, and warning information and all events involved in typhoons in the structure of Tab. 1-4. The detailed information involved in Typhoon Lekima was organized into CSV files according to the table, and then the CSV files were manipulated as a batch.

Table 1: Typhoon State table structure

\begin{tabular}{cccccccc}
\hline No & TIME & Level & X & Y & Pressure & Speed & Direct \\
\hline S1 & $\begin{array}{c}2019 / 8 / 4 \\
17: 00\end{array}$ & TS & 131.5 & 17.1 & 998 & 18 & North \\
\hline
\end{tabular}

Table 2: Warning State Table Structure

\begin{tabular}{|c|c|c|c|c|c|c|c|}
\hline No & TIME & Admin & Wind & Level & Rain_6 & Rain_3 & Mutual \\
\hline 1 & $\begin{array}{c}2019 / 8 / 8 \\
10: 00\end{array}$ & Zhejiang & 12 & L3 & $\ldots$ & $\ldots$ & S33-s38 \\
\hline
\end{tabular}

Table 3: Typhoon Process Table Structure

\begin{tabular}{ccccccc}
\hline No & Name & Start time & End time & Precede & Next & States List \\
\hline \multirow{2}{*}{1} & Birth & $2019 / 8 / 4$ & $2019 / 8 / 6$ & - & Develop & s0--s12 \\
& $17: 00$ & $2: 00$ & & & \\
2 & Develop & $2019 / 8 / 62: 00$ & $2019 / 8 / 7$ & Birth & Keep & s13--s21 \\
\hline \multicolumn{7}{c}{ *Precede and Next is the development relation, States List represents states in } \\
the process.
\end{tabular}

Table 4: Event Table Structure

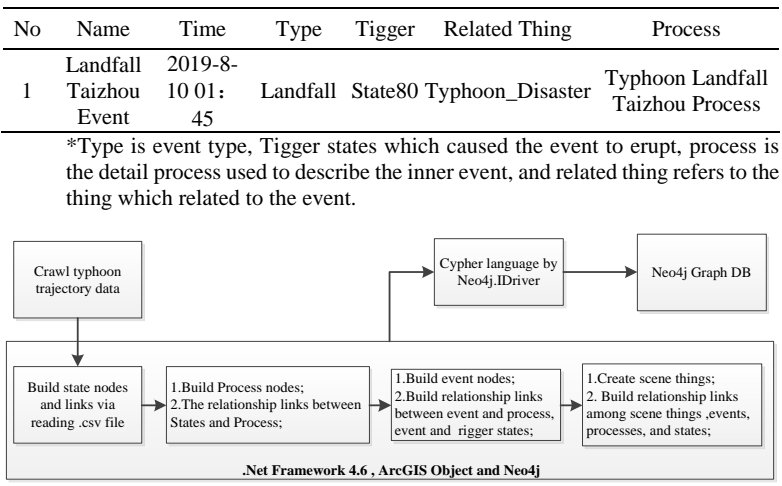

Figure 7: Programming flowchart for typhoon dynamic building

The typhoon dynamic model data, which was constructed by manipulating Cypher language in visual studio 2017 with C\#, was stored in Neo4j graph DB, and the spatial data is developed and organized by ArcObject 10.8. The main steps to implement the construction of the typhoon dynamic model (shown in fig. 7) are as follows: 1) crawl typhoon trajectories from http://typhoon.weath-er.com.cn; 2) Organize the structure of state-process- event geographic scene features from bottom up according to the geographic scene model in this paper, and create scene objects and establish relationships by object-oriented programming method; 3) Store each scene object and relationship to Neo4j database by Neo4j.IDriver; 4) retrieve and analyse the typhoon dynamic model by operating the Neo4j graph database.

\subsection{Data and Software Availability}

The typhoon dynamic model data was stored in Neo4j 4.1.0 which was constructed by manipulating Cypher language in visual studio 2017 with C\#, and the spatial data is developed and organized by ArcObject 10.8 SDK for .net framework.

The Lekima Typhoon information can be obtained from http://typhoon.weather.com.cn/gis/typhoon_p.shtml (last accesses 15.04.2021). The trajectories JSON was obtained from http://d1. weather.com.cn/typhoon/ typhoon_data/2019/1909.json?callback=getData\&_=16 18482419300 (last accessed 10.02.2021), then transformed into lima.csv by python code. Lekima 
Typhoon warning in Zhejiang Province information was extracted from the website (http://www.cma.gov.cn/2011xzt/2019zt/2019tf/20190 808/index_3304.html (last accessed 10.02.2021)). The data mentioned (lima.csv, process.csv and warning.csv) and code used is available in a GitHub repository: https://github.com/heyf2018/DynamicModeOfTyphoon /tree/main/DynamicModel_Typhoon.

\section{Application to Typhoon Event}

Typhoon dynamic model expression was divided into three parts: first, a scene-process-state structure was established for the Lekima typhoon process to simulate the typhoon life cycle dynamically. Then, we integrated the correlation between the typhoon process of Lekima and the typhoon warning pronounced process, and broke the information island situation between each other; finally, we built a geographical scene with typhoon disaster information and typhoon process to facilitate the integration, analysis, and reasoning of multiple information related to typhoon events. The diagram of the spatio-temporal dynamic expression model of typhoon Lekima showed in Fig. 8. Based on the ability of the NeoSemantics plugin in the Neo4j database to support inference on RDF models (Huang et al., 2020), we subsequently demonstrate the ability of spatial inference and complex queries on the typhoon evolution data model built in this paper. We answer the following propositions, which are difficult to answer at once by traditional databases, to demonstrate the dynamic representation and spatial reasoning ability of our model.

Q1: What were the damages caused by the Lekima typhoon in Taizhou?

Query expression in Cypher language:

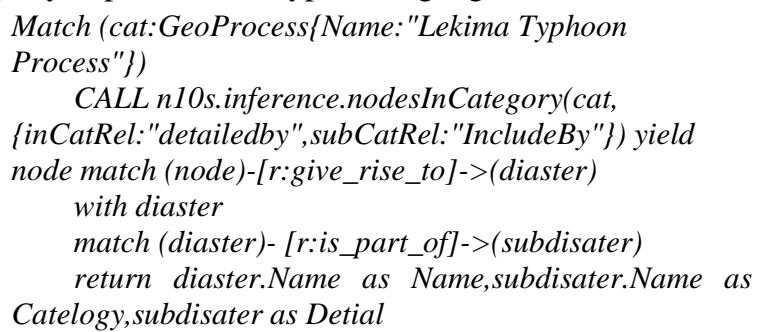

Q2: What was the landfall information for Typhoon Lekima?

Query expression in Cypher language:

Match (cat:GeoProcess\{Name:"Lekima Typhoon Process"\})
CALL n10s.inference.nodesInCategory (cat, \{inCatRel: "registerby",subCatRel:"IncludeBy"\}) yield node return node.Name as Event, node.Time as Time, node.Speed as Speed, node. Level as Level, node. Location as Location

To know the damages information caused by typhoon Lekima, like the description by Q1, the method presented in the paper can obtain the result just by using one query statement. This avoids building lots of tables and joining multiple tables as used to analzes data in traditional spatial data models. Q2 demonstrates the ability to quickly retrieve dynamic information in typhoon dynamic models.

\section{Conclusions and Further Work}

This paper first analysed the relationship between geographic events, geographic processes, and states, and revealed geographic events and processes are the abstractions of geographic changes at different granularity. It was clarified that geographic events are sudden changes of geographic processes or significant geographic processes and geographic processes could describe geographic events in detail. Based on these definitions, a spatio-temporal dynamic expression model was defined which is emphasizing geographic process-events. The implementation of the model avoids creating many tables and then joining multiple tables to get the desired query results as by the traditional data model. It further emphasized the dynamic representation and analysis of the whole life cycle of processes of geographic scene objects. Taking the Lekima typhoon as an example, the usability of the model in expressing typhoon dynamic processes was demonstrated, and a set of methods for building typhoon dynamic processes was provided by Cypher and Neo4j graph database. According to the causal, hierarchical, and developmental relationships constructed by the geographic scene model, we next focus on exploring the potential of the model for spatial inference and prediction for spatio-temporal dynamics.

Funding: This research was funded by the Key Fund of National Natural Science Foundation of China, grant number 41631175; This research was funded by the Key Fund of National Natural Science Foundation of China, grant number 42077003; the National Key Research and Development Program of China, grant number 2017YFB0503500. 
Table 5: Results to question $1(\mathrm{Q} 1)$

\begin{tabular}{|c|c|}
\hline Catalog & Detail \\
\hline $\begin{array}{l}\text { Water Conservancy } \\
\text { Project }\end{array}$ & $\begin{array}{c}\text { \{"Dike breach":15,"Direct economic loss":1.3688,"Damaged reservoir":29,"Damaged embankment":524,"Dike breach } \\
\text { range": } 1.2 \mathrm{~km}, \text {,Damaged embankment range": } 189 \mathrm{~km}\}\end{array}$ \\
\hline Road damage & $\{$ "Rural Bridge":7,"Affecting traffic projects":53,"Damage Highway": 11, ,Rural road":670, \} \\
\hline Social Activities & \{"Seriously Damage the House":9154,"Collapsed House":4107 \} \\
\hline Waterlogging & $\begin{array}{l}\{\text { "waterlogging area WenLin":20\%,"The deepest water Linhai": } 10, \text {,Deepest water Wenlin":2 meters,"The deepest water } \\
\text { Linhai": } 2.5\}\end{array}$ \\
\hline Rainstorm & $\{$ "precipitation":210,"Place":TAIZHOU City $\}$ \\
\hline Power Facilities & $\begin{array}{c}\{\text { "The number of power outages": } 1730000, \text {,The number of } 110 \mathrm{kV} \text { substation": } 27, \text {,The number of } 10 \mathrm{kV} \text { line": } 1684, \text {,The } \\
\text { number of } 220 \mathrm{kV} \text { substation": } 3\}\end{array}$ \\
\hline People & $\{$ ["Affected Population":3723000,"Death":32,"Number of Lost":16 \} \\
\hline Geological Disaster & $\{$ "The number pf collapse":26,"The number of debris flow":5,"The number of landslides":25 \} \\
\hline Business Loss & $\{$ ["Direct Loss": ¥37.5 billion,"The number of enterprises affected": 1870000$\}$ \\
\hline Agriculture Loss & $\{$ \{No Harvest Area":21300 hectares,"The Area Affected": 111000 hectare \\
\hline
\end{tabular}

Table 6: Results to question 2 (Q2)

\begin{tabular}{ccccc}
\hline Event & Time & Speed & Level & Location \\
\hline Lekima Typhoon Loadfall DaLian Event & $2019 / 8 / 101: 45$ & $23 \mathrm{~m} / \mathrm{s}$ & 9 & Huangdao District, Qingdao City \\
Lekima Typhoon Loadfall Taizhou Event & $2019 / 8 / 1120: 00$ & $52 \mathrm{~m} / \mathrm{s}$ & 16 & Chengnan Town, Wenling City \\
\hline
\end{tabular}

\section{References}

Cao, Y., Huang, Y., Chen, J., and Sheng, Y.: Geographic Process Modeling Based on Geographic Ontology, Open Geosciences, 10, 782-796, https://doi.org/10.1515/geo-2018-0061, 2018.

Galton, A.: States, Processes and Events, and the Ontology of Causal Relations, International conference on formal ontology in information systems; International conference on biomedical ontologies, https://doi.org/10.3233/978-1-61499-084-0-279,

Galton, A.: Outline of a Formal Theory of Processes and Events, and Why GIScience Needs One, 2015.

Hornsby, K. S. and Cole, S.: Modeling moving geospatial objects from an event-based perspective, Transactions in GIS, 11, 555-573, https://doi.org/10.1111/j.1467-9671.2007.01060.x, 2007.

Huang, Y., Yuan, M., Sheng, Y., Min, X., and Cao, Y.: Using Geographic Ontologies and Geo-Characterization to Represent Geographic Scenarios, ISPRS international journal of geo-information, 8, 566, https://doi.org/10.3390/ijgi8120566, 2019.

Huang, Z., Jowers, C., Dehghan-Manshadi, A., and Dargusch, M. S.: Smart manufacturing and DVSM based on an Ontological approach, Computers in Industry, 117, 103189, https://doi.org/10.1016/j.compind.2020.103189, 2020.
Li, B., Liu, J., Shi, L., and Wang, Z.: A method of constructing geo-object ontology in disaster system for prevention and decrease, International Symposium on Spatial Analysis, Spatial-Temporal Data Modeling, and Data Mining, 74923I, https://doi.org/10.1117/12.837584,

Lü, G., Batty, M., Strobl, J., Lin, H., Zhu, A.-X., and Chen, M.: Reflections and speculations on the progress in Geographic Information Systems (GIS): a geographic perspective, International journal of geographical information science, 33, 346-367, https://doi.org/10.1080/13658816.2018.1533136, 2019.

Lu, G. N., Chen, M., Yuan, L. W., Zhou, L. C., Wen, Y. N., Wu, M. G., Hu, B., Yu, Z. Y., Yue, S. S., and Sheng, Y. H.: Geographic scenario: a possible foundation for further development of virtual geographic environments, Int. J. Digit. Earth, 11, 356-368, https://doi.org/10.1080/17538947.2017.1374477, 2018.

Uschold, M.: Ontology-driven information systems: Past, present and future, Proceedings of the 2008 conference on Formal Ontology in Information Systems: Proceedings of the Fifth International Conference (FOIS 2008), 3-18,

Zhao, L. and Li, C.: Ontology based opinion mining for movie reviews, International Conference on Knowledge Science, Engineering and Management, 204-214, https://doi.org/10.1007/978-3-642-10488-6_22, 


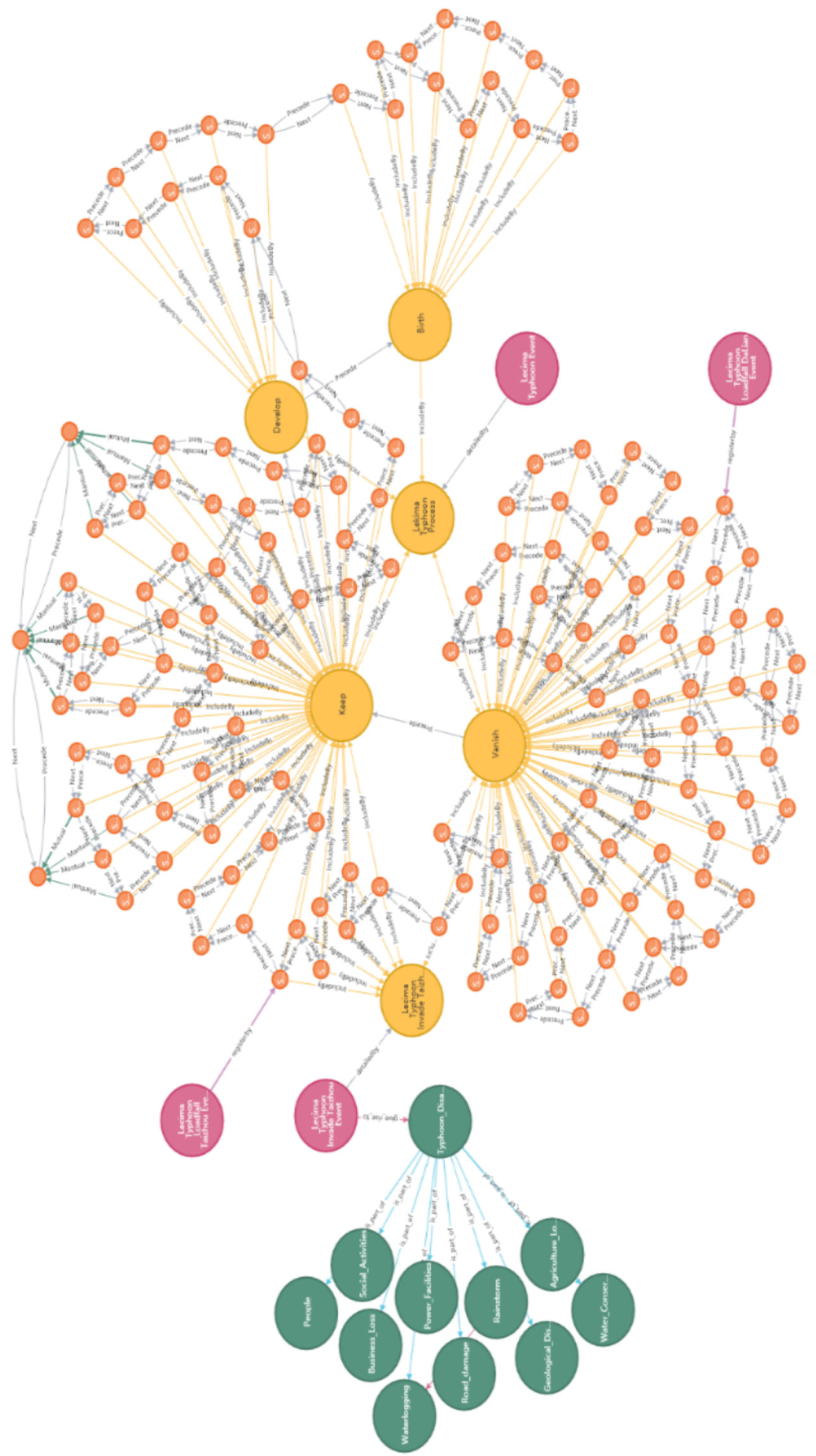

Figure 8: Geographical Dynamic model of Typhoon Lekima implemented in Neo4J 